\title{
Disruptive Mood Dysregulation Disorder (DMDD)
}

\section{Eman A Zaky*}

Department of Pediatrics, Faculty of Medicine, Ain Shams University, Egypt

\begin{abstract}
In order to address the concerns about the potential for the over-diagnosis of and treatment for bipolar disorder in children, a new diagnosis, disruptive mood dysregulation disorder (DMDD) has been recently added to the depressive disorders for children up to 12 years of age in DSM 5. It refers to the presentation of children with persistent irritability and frequent episodes of extreme behavioral dyscontrol. Such children were found to typically develop unipolar depressive disorders or anxiety disorders rather than bipolar disorders as they mature into adolescence and adulthood. Whether this new diagnosis will also lead to a more judicious use of psychotropic medications and increased utilization of behavioral, psychosocial, and family interventions remains to be seen.
\end{abstract}

Keywords: Disruptive mood dysregulation disorder (DMDD); Bipolar disorder (BD); Oppositional defiant disorder (ODD); DSM 5

\section{Disruptive Mood Dysregulation Disorder (DMDD)}

Disruptive Mood Dysregulation Disorder (DMDD) refers to the presentation of children with persistent irritability and frequent episodes of extreme behavioral dyscontrol. It has been recently added to the depressive disorders for children up to 12 years of age in DSM 5 [1].

\section{What is disruptive mood dysregulation disorder (DMDD)?}

Far beyond temper tantrums, DMDD is characterized by severe and recurrent temper outbursts that are grossly out of proportion in intensity or duration to the situation. These occur, on average, three or more times each week for one year or more. Between outbursts, children with DMDD display a persistently irritable or angry mood, most of the day and nearly every day, that is observable by parents, teachers, or peers. A diagnosis requires the above symptoms to be present in at least two settings (at home, at school, or with peers) for 12 or more months, and symptoms must be severe in at least one of these settings. During this period, the child must not have gone three or more consecutive months without symptoms. The onset of symptoms must be before age 10 , and a DMDD diagnosis should not be made for the first time before age 6 or after age 18 [1].

\section{DMDD, a new diagnosis in DSM 5, why?}

Researchers identified many problems in diagnosis of childhood bipolar disorder that necessitated the addition of DMDD as a new diagnostic category in DSM 5. These problems were reflected by the fact that diagnosis of bipolar disorder (BD) in children went up 4000\% from 1994 to 2003. Such huge increase in BD diagnosis was thought to be caused by "loose" translation of DSM-IV (1994) [2] criteria for BD when applied to children. So, researchers seriously considered changing diagnostic criteria for children suffering from longitudinal course of severe and recurrent temper outbursts that are grossly out of proportion in intensity or duration to the situation but they concluded that original BD criteria should stand. Accordingly, the newly added DMDD was developed in DSM V to identify children not meeting the diagnostic criteria of BD yet having significant impairment. Furthermore, "Bipolar Disorder Not Otherwise Specified" category which was commonly applied to children not meeting BD full criteria has been removed in DSM 5.

\section{DSM 5 diagnostic criteria of DMDD}

A. Severe recurrent temper outbursts manifested verbally (e.g., verbal rages) and/or behaviorally (e.g., physical aggression toward people or property) that are grossly out of proportion in intensity or duration to the situation or provocation.

B. The temper outbursts are inconsistent with developmental level.

C. The temper outbursts occur, on average, three or more times per week.

D. The mood between temper outbursts is persistently irritable or angry most of the day, nearly every day, and is observable by others (e.g., parents, teachers, peers).

E. Criteria A-D have been present for 12 or more months. Throughout that time, the individual has not had a period lasting 3 or more consecutive months without all of the symptoms in Criteria A-D.

F. Criteria A and D are present in at least two of three settings (i.e., at home, at school, with peers) and are severe in at least one of these.

G. The diagnosis should not be made for the first time before age 6 years or after age 18 years. H. By history or observation, the age at onset of Criteria A-E is before 10 years.

I. There has never been a distinct period lasting more than 1 day during which the full symptom criteria, except duration, for a manic or hypomanic episode have been met. Note: Developmentally appropriate mood elevation, such as that occurs in the context of a highly positive event or its anticipation, should not be considered as a symptom of mania or hypomania.

J. The behaviors do not occur exclusively during an episode of major

*Corresponding author: Eman Ahmed Zaky, Department of Pediatrics, Faculty of Medicine, Ain Shams University, Head of Child Psychiatry Clinic, Children's Hospital, Ain Shams University, Cairo, Egypt, E-mail: emanzaky@med.asu.edu.eg

Received: September 22, 2015; Accepted: September 25, 2015; Published: November 15, 2015

Citation: Zaky EA (2015) Disruptive Mood Dysregulation Disorder (DMDD). Clin Depress 1: e102

Copyright: () 2015 Zaky EA. This is an open-access article distributed under the terms of the Creative Commons Attribution License, which permits unrestricted use, distribution, and reproduction in any medium, provided the original author and source are credited. 
depressive disorder and are not better explained by another mental disorder (e.g., autism spectrum disorder, posttraumatic stress disorder, separation anxiety disorder, persistent depressive disorder [dysthymia]).

Note: This diagnosis cannot coexist with oppositional defiant disorder, intermittent explosive disorder, or bipolar disorder, though it can coexist with others, including major depressive disorder, attention deficit/ hyperactivity disorder, conduct disorder, and substance use disorders. Individuals whose symptoms meet criteria for both disruptive mood dysregulation disorder and oppositional defiant disorder should only be given the diagnosis of disruptive mood dysregulation disorder. If an individual has ever experienced a manic or hypomanic episode, the diagnosis of disruptive mood dysregulation disorder should not be assigned.

$\mathrm{K}$. The symptoms are not attributable to the physiological effects of a substance or to another medical or neurological condition.

\section{Prevalence}

The prevalence of DMDD in the community is still unclear but based on rates of chronic and severe persistent irritability, which is the core feature of the disorder, the overall 6 months to 1 year period prevalence of DMDD among children and adolescents probably falls in the $2-5 \%$ range. Rates are expected to be higher in males and school aged children compared to females and adolescents. Gender impact in DMDD is unlike the equal gender prevalence in $\mathrm{BD}[1]$.

\section{Functional Consequences of DMDD}

Like children with pediatric $\mathrm{BD}$ or other mental illnesses, those with DMDD exhibit both commonalities and differences in information processing deficits as face emotion labeling deficits and perturbed decision making and cognitive control. On the other hand, there is an evidence for DMDD specific dysfunction such as during tasks assessing attention deployment in response to emotional stimuli. Suicidal behavior and aggression have been documented among patients with DMDD as one of its severe functional consequences. Chronic and severe irritability seen in DMDD are usually associated with marked disruption in a child's family and peer relationships as well as in school performance.

\section{Differential Diagnosis of DMDD}

The diagnosis of DMDD must be made while considering the presence or absence of multiple other conditions as oppositional defiant disorder (ODD) and bipolar disorders (BD) as chronically irritable children and adolescents typically present with complex histories. In order to differentiate DMDD from any other possible differential diagnosis, careful assessment is crucial.

ODD is an ongoing pattern of anger-guided disobedience, hostilely defiant behavior toward authority figures that goes beyond the bounds of normal childhood behavior. While some of its symptoms may overlap with the criteria for DMDD, the symptom threshold for DMDD is higher since the condition is considered more severe. To avoid any artificial comorbidity of the two disorders, it is recommended that children who meet criteria for both ODD and DMDD should only be diagnosed with DMDD [2].

On the other hand, $\mathrm{BD}$ also has similar symptoms and unfortunately, clinicians may have been assigning a $\mathrm{BD}$ diagnosis to these severely irritable youth to ensure their access to treatment resources and services. The central feature that differentiate DMDD from $\mathrm{BD}$ is the longitudinal course of DMDD as children suffering from the latter's characteristically have behaviors that do not present in an episodic way as is the case with $\mathrm{BD}[1]$.

Concerning the differentiation of autistic disorder from DMDD, it just could be done using soft exclusionary criteria in DMDD best expressed as "cannot better be explained by Autistic Disorder" and non overlap of symptoms but yet there is a little explanation of how these two disorders interact [1].

\section{Possible Comorbid Conditions}

Comorbid conditions that can coexist with DMDD include major depressive disorder, Attention Deficit/Hyperactivity Disorder, conduct disorder, substance use disorders, and anxiety disorders [1].

\section{Treatment}

DMDD is best treated with the help of psychotherapy and occasionally medications. Medication is ideally not suitable for children that are very young and psychotherapy is ideally the best approach in such children after exclusion of other illnesses or disabilities that may be the root cause of the disruptive mood dysregulation disorder. Normal temper tantrums no matter how severe tend to lessen in frequency and duration as the child grows older while DMDD requires psychiatric treatment.

\section{Criticism and Future scope}

Although the validity and specificity of the new DMDD diagnosis remain in question by many clinicians, the hope is that the documented rise in bipolar disorder diagnoses for non episodic mood dysregulation and aggression with prepubertal onset may decrease. Whether this new diagnosis will also lead to a more judicious use of psychotropic medications and increased utilization of behavioral, psychosocial, and family interventions remains to be seen, but the addition of DMDD in DSM-5 will hopefully encourage such research. Also, defining this disorder as a distinct condition is expected to have a considerable impact on clinical practice and thus its management.

To summarize, one can say that the unique features of DMDD necessitated a new diagnosis in DSM 5 to ensure that children affected by this disorder will get the proper clinical help they need with improvement of their diagnosis, care, and quality of life. Finally, it is always worthy to remember that the road to mental health begins with an accurate diagnosis.

\section{References}

1. DSM 5 (2013) American Psychiatric Association: Diagnostic and Statistical Manual of Mental Disorders, 5th Edition. Washington, DC, American Psychiatric Association, pp: 155-160.

2. DSM IV (1994) American Psychiatric Association: Diagnostic and Statistical Manual of Mental Disorders, 4th Edition. Washington, DC, American Psychiatric Association. 